\title{
Genomic Biosurveillance Detects A Sexual Hybrid in the Sudden Oak Death Pathogen
}

\section{Richard Hamelin ( $\nabla$ richard.hamelin@ubc.ca )}

University of British Columbia https://orcid.org/0000-0003-4006-532X

\section{Guillaume Bilodeau}

Canadian Food Inspection Agency https://orcid.org/0000-0003-1766-229X

\section{Renate Heinzelmann}

Faculty of Forestry, The University of British Columbia

\section{Kelly Hrywkiw}

Faculty of Forestry, The University of British Columbia

\section{Arnaud Capron}

Faculty of Forestry, The University of British Columbia

\section{Erika Dort}

Faculty of Forestry, The University of British Columbia

\section{Angela Dale}

FPInnvations

\section{Emilie Giroux}

Canadian Food Inspection Agency

\section{Nick Carleson}

Oregon State University

\section{Niklaus Grünwald}

USDA ARS https://orcid.org/0000-0003-1656-7602

\section{Nicolas Feau}

Faculty of Forestry, The University of British Columbia

\section{Stacey Kus}

FPInnovations

\section{Article}

Keywords: Invasive exotic pathogens, Phytophthora ramorum, nucleotide polymorphism, biosurveillance.

Posted Date: October 28th, 2021

DOI: https://doi.org/10.21203/rs.3.rs-699860/v1 
License: (c) (i) This work is licensed under a Creative Commons Attribution 4.0 International License. Read Full License 


\section{Abstract}

Invasive exotic pathogens pose a threat to trees and forest ecosystems worldwide ${ }^{1}$, hampering the provision of essential ecosystem services such as carbon sequestration and water purification ${ }^{2}$. Hybridization is a major evolutionary force that can drive the emergence of pathogens ${ }^{3}$. Phytophthora ramorum, an emergent pathogen that causes the sudden oak and larch death, spreads as reproductively isolated divergent clonal lineages. Sexual recombination has never been reported in this pathogen under natural conditions and laboratory crosses have yielded unfit progenies, suggesting postzygotic barriers to hybridization. Here we report the discovery in a plant nursery of novel variants of $P$. ramorum that are the result of homoploid hybridization via sexual recombination between North American and European lineages of the pathogen. We show that these hybrids are viable, can infect plants and produce spores for long-term survival and propagation. Genome sequencing revealed novel genotypic combinations, not present in the parental lineages, at 54,515 single nucleotide polymorphism loci. More than 6000 of the novel genotypes at these loci are predicted to have a functional impact in genes associated with host infection, including effectors, carbohydrate-active enzymes and proteases. We also observed post-meiotic mitotic recombination that could generate additional genotypic and phenotypic variation and contribute to homoploid hybrid speciation. Our study highlights the importance of plant pathogen biosurveillance to detect novel variants and inform management and control.

\section{Main}

Emerging infectious diseases pose a threat to trees and forest ecosystems worldwide ${ }^{1}$. Outbreaks of invasive exotic pathogens can reduce the ability of forests to provide essential ecosystem services such as carbon sequestration and water purification ${ }^{2}$. Hybridization between species or individuals from divergent lineages can drive the emergence of novel pathogens by generating new genotypic and phenotypic combinations that can enhance fitness and allow colonization of new niches ${ }^{3-8}$. This can be important for invasive plant pathogens that propagate asexually via clonal lineages, because the new genotypic combinations can generate novel lifestyles or host shifts ${ }^{5}$ and purge deleterious mutations accumulated via Muller's ratchet ${ }^{9-11}$. Phytophthora ramorum is an exotic plant pathogen that emerged in the 1990s and is regulated and targeted for eradication in Europe and North America. It can attack over 125 plant species and is responsible for the sudden oak death in California and Oregon ${ }^{12}$ and sudden larch death in the $\mathrm{UK}^{13}$ and France ${ }^{14}$. The pathogen comprises divergent clonal lineages ${ }^{15}$ that are reproductively isolated and confined to North America (NA1, NA2) or Europe (EU2), one that is broadly distributed in Europe and the West Coast of North America (EU1) ${ }^{16,17}$, and additional lineages recently described in $\mathrm{Asia}^{18}$. Recombination can only take place between individuals of opposite mating types and was demonstrated in laboratory conditions between the EU1 (mating type A1) and NA1 (mating type A2) lineages ${ }^{19-21}$ but the progeny displayed aberrant genotypic and phenotypic variation. Sexual recombination between or within lineages has not been observed in natural conditions in the invasive range of the pathogen ${ }^{17}$. 


\section{Hybridization between divergent clonal lineages}

We discovered hybrids between the EU1 and NA2 clonal lineages of $P$. ramorum in a North American nursery, where these lineages co-occurred. The qPCR lineage genotyping pattern of isolates 16-237-021 and 16-284-032, collected on infected rhododendron plants, do not match the pattern of the known clonal $P$. ramorum lineages in North America and Europe (Table S1). We sequenced the genomes of $95 P$. ramorum isolates (Table S2) from Europe and North America and mapped the reads onto the reference genome, yielding 450,656 single nucleotide polymorphisms (SNP). Of the 31,047 SNPs that were homozygous for different alleles in the EU1 and NA2 lineages, 96.6\% were heterozygous in isolates 16237-021 and 16-284-032 (Table S3). A principal component analysis of the SNPs placed the two putative hybrids between the EU1 and NA2 lineages and ancestry estimation assigned them with equal probability to those putative parental lineages (Fig. 1A, B). A phylogenetic network analysis placed the hybrid samples in a branch with shared reticulations with lineages EU1 and NA2, the pattern expected for recombination (Fig. 1C).

\section{First-generation homoploid hybrids generated via sexual recombination}

The hybrids are likely homoploid (without a change in chromosome copy number) and result from a first generation (F1) recombination event. Analysis of phased genomic regions revealed two distinct haplotypes in the hybrids, each clustering either with a NA2 or an EU1 haplotype (Fig. 2A, B, C). The contribution of the putative parents to the nuclear genome of the hybrids was equal (Z-score $=8.78 \pm 0.24$; $p<0.0001$; Table S4) and they were assigned to a simulated EU1 x NA2 population with $\geq 0.999$ probability (assignment to all other populations < 1.0e-04; Fig. S1A). A neighbor-joining tree clustered the hybrid samples with simulated first-generation hybrids but not with simulated populations backcrossed to each parental lineage (Fig. S2). The two hybrid samples share genotypes at $99.8 \%$ of the SNP loci and are thus likely clones derived from a single hybridization event, via meiotic recombination, followed by clonal propagation. The observed genotypic combinations in the hybrids and the parental lineages support the hypothesis of meiotic recombination (except for some excess of homozygosity observed in the hybrids [Table S3; see below]) but not of somatic recombination (Table S3). The NA2 lineage likely acted as the "female" parent. We mapped the sequence reads of all 95 sequenced genomes to the mitochondrial genome of $P$. ramorum and retrieved their mitochondrial haplotypes. The EU1 x NA2 hybrids share mitochondrial haplotypes (comprising 103 polymorphic sites) with members of the NA2 lineage (Fig. 2D), indicating uniparental transmission of the mitochondrial genome ${ }^{19}$.

\section{Predicted functional impact of hybridization}

The hybridization between $P$. ramorum lineages that diverged approximately 1 million years ago ${ }^{15,22}$ could impact fitness. These lineages differ in several traits, including growth, sporulation and aggressiveness during host infection ${ }^{18,23}$. The growth rate of the hybrid isolates was intermediate 
between the parental lineages but significantly higher than the EU1 parent ( $p<0,01$; Fig. 3D). The hybrids can produce both chlamydospores and sporangia (Fig. 3A, B \& C), spores that are important for survival and spread. The hybrids were infectious on rhododendron, a common host, and caused lesions with sizes that overlapped with those of the other lineages (Fig. 3E). Previous studies with artificial crosses between $P$. ramorum lineages produced progeny with reduced viability but that exhibited a broad range of pathogenicity ${ }^{19}$. This could be explained by transgressive trait variation, a common outcome of hybridization in plants, where hybrid phenotypes exceed those of the parents, contributing to ecological niche divergence ${ }^{24}$. The hybrid $P$. ramorum have novel genotypes, not observed in the parental lineages, at 54,515 SNPs that are either homozygous for different alleles in the parental lineages but heterozygous in the hybrid, or heterozygous in both parental lineages but homozygous in the hybrid (Table S3). Of those, 6,736 are non-synonymous mutations and 6,752 are predicted to have a moderate (e.g., nonsynonymous mutations) to high (e.g., stop codons, frameshifts) impact (Tables S5 and S6). Several of the genes that have novel genotypic combinations in the hybrids are associated with pathogenicity $25-27$, including 51 RxLR and crinkler-like effectors ${ }^{27}, 79$ carbohydrate active enzymes (CAZy) and 11 peptidases (Table S7). A single amino acid polymorphism in a pathogen effector was recently shown to expand its binding spectrum ${ }^{28}$, thus the observed novel genotypes in the $P$. ramorum hybrid could impact diversification and adaptation in this pathogen.

\section{Mitotic recombination generates additional variation}

Mitotic recombination in the hybrid could provide additional genotypic and phenotypic variation in the pathogen population. We found 1,049 SNPs that were homozygous for different alleles in the parental lineages and also homozygous for one of those alleles in the hybrids, an unexpected pattern for sexual recombinants (Table S3). We observed that $90 \%$ of those SNPs were distributed in stretches of 2 to 82 (median $=4.0$ ) contiguous homozygous positions (runs of homozygosity; ROH) in the hybrid (Fig. S3). These $\mathrm{ROH}$ were in gene-poor regions enriched in transposable elements where RxLR effectors and putative avirulence factors are present (Table S8). Mitotic recombination was shown to accelerate adaptation in fungi ${ }^{29}$ and appears to be an important mechanism fueling evolution in $P$. ramorum, producing $\mathrm{ROH}$ in all lineages ${ }^{22}$. Virulence differences and adaptation to environmental changes such as exposure to oxidative or heat stress and antifungal drugs have been associated with $\mathrm{ROH}$ in other species $^{30}$ and could increase genotypic and phenotypic diversity and increase divergence between the $P$. ramorum lineages and the new hybrid.

\section{Discussion and outlook}

The discovery of hybridization among lineages of $P$. ramorum should cause concerns in the plant and forest health communities. Hybridization provides a source of new genetic variation ${ }^{31}$ upon which natural selection can act to modify traits such as pathogenicity and transmission ${ }^{32}$. Selective forces could create the conditions for homoploid hybrid speciation ${ }^{33}$ and the emergence of novel lineages with distinct traits. This could modify the epidemiological parameters of the outbreak. The oospores that are the product of 
sexual reproduction can survive adverse conditions such as drought and freeze and remain viable in the soil for years, making it possible for the pathogen to survive in the absence of a host ${ }^{34}$ thereby increasing the risk of accidental propagation. In the potato famine pathogen, Phytophthora infestans, sexual recombination became possible when lineages of the opposite mating type migrated from the center of

origin $^{35-38}$. This increased diversity contributed to the emergence of novel virulence combinations ${ }^{39}$, coinciding with the appearance of lineages that were resistant to fungicides ${ }^{38}$ and outbreaks that started earlier in the season, generating an increased disease risk ${ }^{40}$.

So far, the $P$. ramorum EU1 x NA2 hybrid was only found in a single nursery in British Columbia, where the pathogen has not spread to natural forests. The disease was apparently eradicated in that nursery, preventing propagation of the hybrid. But in Oregon, where lineages of $P$. ramorum with different mating types co-occur in natural forests ${ }^{42,43}$, the spread of a hybrid lineage could be more difficult to contain. Our study highlights the importance of genomic biosurveillance for the detection of novel plant pathogen variants and hybrids to inform mitigation strategies ${ }^{44}$.

\section{Methods}

\section{Sample collection and genomic characterization}

We obtained isolates of $P$. ramorum from nurseries in BC, Canada between 1995 and 2017 (Table S2). Genome sequences were obtained either using Illumina Hiseq or lon Torrent S5. Sequence reads were mapped onto the $P$. ramorum NA1 reference genome PR-102_v3.1

(https://doi.org/10.1101/2021.06.23.449625; BioProject PRJNA738483). We also retrieved previously sequenced genomes from Canada ${ }^{22}$, the $\mathrm{US}^{45}$ and Europe $\mathrm{e}^{22,46}$ at NCBI

(https://www.ncbi.nlm.nih.gov/bioproject/) for a total of 95 P. ramorum genomes. Variant Call Format (VCF) files were obtained for all genomes as described in the supplementary materials. Mitochondrial haplotypes were obtained by mapping the reads on the $P$. ramorum reference genome ( $\operatorname{Pr} 102, \mathrm{NCBI}$ accession: NC_009384.1 ${ }^{47}$ ) and variants were called.

\section{Population genomics analyses}

Population structuring and genetic connectivity of the unknown isolates with the four lineages of $P$. ramorum was evaluated using Principal Component Analysis (PCA) with SNPrelate ${ }^{48}$. Linkage disequilibrium-pruning was applied on the VCF dataset of 450,656 SNP loci to subsample 3,780 markers with reduced linkage $\left(R^{2}<0.20\right)$. Ancestry estimation was conducted using Admixture among the $P$. ramorum lineages with the full SNP set with $\mathrm{K}=4$, the number of lineages found in North American and Europe in $P$. ramorum. To assess reticulated relationships between $P$. ramorum individuals a neighbor-net phylogenetic network was reconstructed using SplitsTree v4.12.8 ${ }^{49}$ with pairwise Nei's genetic distances with the R package StAMPP ${ }^{50}$.

\section{Genome-wide hybridization detection and gene flow simulations}


We used the function hybridization in the Adegenet R package to simulate $10 \mathrm{~F} 1$ hybrids between NA2 and EU1 and 10 backcrosses of the hybrid to the EU1 or NA2 parent. We generated a pairwise distance matrix with the dist function and obtained an NJ tree to visualize the position of the observed samples and simulation of hybrids and introgressants. We used the program $\mathrm{HyDe}^{51}$ to perform a genome-wide test of interspecific hybridization between $P$. ramorum lineages. HyDe considers a four-taxa network consisting of an outgroup and a triplet of ingroup taxa. The distribution of SNP site patterns in the triplet is used to infer a hybrid ingroup lineage that with a probability $y$ is sister to one lineage and with probability $1-y$ is sister to the other lineage. Under the null hypothesis that admixture is absent, the expected value of $y$ is zero. HyDe testing was performed with the 'run_hyde_mp.py' script of the HyDe package on all possible triplet combinations of putative parents-hybrid with NA1, NA2, EU1 and the 16237-021 and 16-284-032 isolates (i.e. 12 triplets tested) while EU2 samples were used as outgroup. To obtain average $y$-values with standard deviations, the full dataset of 450,656 SNP loci was subsampled 500 times for 10,000 random loci. The Z-statistic was used to test for significance of the y-values, and $p$ values were adjusted for multiple testing using Bonferroni adjustment $(\alpha=0.05 / 12=0.0041)$.

\section{Haplotype phylogenetic network reconstruction}

Phased haplotypes were obtained by using short genome regions with physical phasing when two or more variants co-occur on the same sequencing read using WhatsHap ${ }^{52}$. We tested the 15,265 genes annotated in the $P$. ramorum NA1 reference genome PR-102_v3.1 to identify genes containing at least 10 phased SNP loci in the two hybrid samples and three samples from each $P$. ramorum lineage. A FASTA format sequence alignment file containing two haplotype sequences for each $P$. ramorum samples (including the two hybrids) was then reconstructed as follows: the single nucleotide haplotypes predicted from a phased genotype were coded with their respective allele; the single nucleotide haplotypes predicted from an unphased genotype were coded as missing data. Finally, sequence alignments were collapsed to unique haplotypes within each $P$. ramorum lineage. Maximum likelihood $(\mathrm{ML})$ gene trees were inferred using RAxML ${ }^{53}$ under the GTR model with a GAMMA parameter. Bootstrap support at nodes was determined by 1,000 iterations.

\section{Functional impact and phenotypic characterization}

The potential impact of the SNPs was categorized with regard to types and function with SnpEff (Version 4.3) with default settings. We used the reference genome (PR-102_v3.1) and the gff file to build the annotation database. Growth and morphological characterization was performed on carrot agar $(\mathrm{CA})^{18}$ for the two hybrids and selected isolates of the EU1, NA2, EU2 and NA1 lineages.

\section{Declarations}

\section{Contributions}


$\mathrm{RCH}, \mathrm{GJB}, \mathrm{NF}, \mathrm{AC}$ and RH conceived the project, obtained the samples and performed the genome analyses.

$\mathrm{KH}, \mathrm{ED}, \mathrm{ALD}, \mathrm{AC}$, SK and EG performed the phenotyping.

NCC and NJG generated a reference genome that was used for mapping and analyses and provided annotations.

$\mathrm{RCH}, \mathrm{NF}, \mathrm{RH}$ and GJB wrote the manuscript and the supplementary information with input from all other authors.

\section{Acknowledgments}

This work was supported by Genome Canada, Genome British Columbia, Genome Quebec, Natural Resources, the Canadian Food Inspection Agency and FPInnovations through the Large Scale Applied Research Project (LSARP 10106. The authors acknowledge the help of Debbie Shearlaw, Miranda Newton and Marie-Claude Gagnon from CFIA for technical assistance.

\section{References}

1. Fisher, M. C. et al. Emerging fungal threats to animal, plant and ecosystem health. Nature 484, 186194 (2012).

2. Boyd, I. L., Freer-Smith, P. H., Gilligan, C. A. \& Godfray, H. C. J. The consequence of tree pests and diseases for ecosystem services. Science 342, 1235773-1235773 (2013).

3. Brasier, C. M. The rise of the hybrid fungi. Nature 405, 134-135 (2000).

4. Stukenbrock, E. H. The role of hybridization in the evolution and emergence of new fungal plant pathogens. Phytopathology 106, 104-112 (2016).

5. Mixão, V. \& Gabaldón, T. Hybridization and emergence of virulence in opportunistic human yeast pathogens. Yeast 35, 5-20 (2018).

6. Hessenauer, P. et al. Hybridization and introgression drive genome evolution of Dutch elm disease pathogens. Nature Ecology \& Evolution 1-13 (2020).

7. Pryszcz, L. P. et al. The Genomic Aftermath of Hybridization in the Opportunistic Pathogen Candida metapsilosis. PLoS Genet 11, e1005626 (2015).

8. Menardo, F. et al. Hybridization of powdery mildew strains gives rise to pathogens on novel agricultural crop species. Nature Genetics 48, 201-205 (2016).

9. Haag, C. R. \& Roze, D. Genetic load in sexual and asexual diploids: segregation, dominance and genetic drift. Genetics 176, 1663-1678 (2007).

10. Agrawal, A. F. \& Whitlock, M. C. Mutation load: the fitness of individuals in populations where deleterious alleles are abundant. Annual Review of Ecology, Evolution, and Systematics 43, 115-135 
(2012).

11. Lynch, M., Bürger, R., Butcher, D. \& Gabriel, W. The mutational meltdown in asexual populations. Journal of Heredity 84, 339-344 (1993).

12. Rizzo, D. M. \& Garbelotto, M. Sudden oak death: endangering California and Oregon forest ecosystems. Frontiers in Ecology and the Environment 1, 197-204 (2003).

13. Brasier, C. \& Webber, J. Plant pathology: Sudden larch death. Nature 466, 824-825 (2010).

14. Schenck, N. et al. First report of Phytophthora ramorum causing Japanese larch dieback in France. Plant disease 102, 2045-2045 (2018).

15. Goss, E., Carbone, I. \& Grünwald, N. Ancient isolation and independent evolution of the three clonal lineages of the exotic sudden oak death pathogen Phytophthora ramorum. (2009).

16. Grünwald, N. J. et al. Standardizing the nomenclature for clonal lineages of the sudden oak death pathogen, Phytophthora ramorum. Phytopathology 99, 792-795 (2009).

17. Grünwald, N. J., LeBoldus, J. M. \& Hamelin, R. C. Ecology and Evolution of the Sudden Oak Death Pathogen Phytophthora ramorum. Annual Review of Phytopathology 57, (2019).

18. Jung, T. et al. The Destructive Tree Pathogen Phytophthora ramorum Originates from the Laurosilva Forests of East Asia. JoF 7, 226 (2021).

19. Boutet, X., Vercauteren, A., Heungens, K., Laurent, F. \& Chandelier, A. Oospores progenies from and Phytophthora ramorum. Fungal Biology 114, 369-378 (2010).

20. Brasier, C. \& Kirk, S. Production of gametangia by Phytophthora ramorum in vitro. Mycological research 108, 823-827 (2004).

21. Vercauteren, A. et al. Aberrant genome size and instability of Phytophthora ramorum oospore progenies. Fungal Genetics and Biology 48, 537-543 (2011).

22. Dale, A. L. et al. Mitotic recombination and rapid genome evolution in the invasive forest pathogen Phytophthora ramorum. mBio 10, e02452-18 (2019).

23. Elliott, M. et al. Phenotypic differences among three clonal lineages of Phytophthora ramorum. Forest Pathology 41, 7-14 (2011).

24. Rieseberg, L. H., Archer, M. A. \& Wayne, R. K. Transgressive segregation, adaptation and speciation. Heredity 83, 363-372 (1999).

25. Giraldo, M. C. \& Valent, B. Filamentous plant pathogen effectors in action. Nat Rev Micro 11, 800814 (2013).

26. Dodds, P. N. et al. Effectors of biotrophic fungi and oomycetes: pathogenicity factors and triggers of host resistance. New Phytologist 183, 993-1000 (2009).

27. Kamoun, S. A catalogue of the effector secretome of plant pathogenic oomycetes. Annual Reviews (2006).

28. Bentham, A. R. et al. A single amino acid polymorphism in a conserved effector of the multihost blast fungus pathogen expands host-target binding spectrum. http://biorxiv.org/lookup/doi/10.1101/2021.03.15.435478 (2021) doi:10.1101/2021.03.15.435478. 
29. Schoustra, S. E., Debets, A. J., Slakhorst, M. \& Hoekstra, R. F. Mitotic recombination accelerates adaptation in the fungus Aspergillus nidulans. PLoS Genet 3, e68 (2007).

30. Forche, A. et al. Stress alters rates and types of loss of heterozygosity in Candida albicans. mBio $\mathbf{2}$, (2011).

31. Barton, N. H. The role of hybridization in evolution. Molecular ecology 10, 551-568 (2001).

32. King, K. C., Stelkens, R. B., Webster, J. P., Smith, D. F. \& Brockhurst, M. A. Hybridization in parasites: consequences for adaptive evolution, pathogenesis, and public health in a changing world. PLoS pathogens 11, e1005098 (2015).

33. Yakimowski, S. B. \& Rieseberg, L. H. The role of homoploid hybridization in evolution: A century of studies synthesizing genetics and ecology. American Journal of Botany 101, 1247-1258 (2014).

34. Turkensteen, L., Flier, W., Wanningen, R. \& Mulder, A. Production, survival and infectivity of oospores of Phytophthora infestans. Plant Pathology 49, 688-696 (2000).

35. Gavino, P. D. et al. Implications of sexual reproduction for phytophthora infestans in the United States: generation of an aggressive lineage. Plant Disease 84, 731-735 (2000).

36. Goodwin, S. B., Cohen, B. A., Deahl, K. L. \& Fry, W. E. Migration from northern Mexico as the probable cause of recent genetic changes in populations of Phytophthora infestans in the United States and Canada. Phytopathology 84, 553-558 (1994).

37. Goss, E. M. et al. The Irish potato famine pathogen Phytophthora infestans originated in central Mexico rather than the Andes. Proceedings of the National Academy of Sciences 111, 8791-8796 (2014).

38. Fry, W. et al. Five reasons to consider Phytophthora infestans a reemerging pathogen. Phytopathology 105, 966-981 (2015).

39. Drenth, A., Tas, I. C. \& Govers, F. DNA fingerprinting uncovers a new sexually reproducing population of Phytophthora infestans in the Netherlands. European Journal of Plant Pathology 100, 97-107 (1994).

40. Hannukkala, A., Kaukoranta, T., Lehtinen, A. \& Rahkonen, A. Late-blight epidemics on potato in Finland, 1933-2002; increased and earlier occurrence of epidemics associated with climate change and lack of rotation. Plant pathology 56, 167-176 (2007).

41. Zhan, J., Mundt, C. C. \& McDonald, B. A. Sexual reproduction facilitates the adaptation of parasites to antagonistic host environments: evidence from empirical study in the wheat-Mycosphaerella graminicola system. International journal for parasitology 37, 861-870 (2007).

42. LeBoldus, J. et al. First report of Phytophthora ramorum lineage EU1 infecting Douglas fir and Grand fir in Oregon. Plant Disease 102, 455-455 (2018).

43. Grünwald, N. et al. First report of the EU1 clonal lineage of Phytophthora ramorum on tanoak in an Oregon forest. Plant Disease 100, 1024-1024 (2016).

44. Hamelin, R. C. \& Roe, A. D. Genomic biosurveillance of forest invasive alien enemies: A story written in code. Evolutionary Applications 13, 95-115 (2020). 
45. Yuzon, J., Rizzo, D. M., Tripathy, S., Kasuga, T., \& others. Resequencing of the Phytophthora ramorum genome to characterize genetic variation and population dynamics of the invasive pathogen. in Proceedings of the sudden oak death sixth science symposium. Gen. Tech. Rep. GTR-PSW-255. Albany, CA: US Department of Agriculture, Forest Service, Pacific Southwest Research Station: 105106. 105-106 (2017).

46. Turner, J. et al. Genome sequences of 12 isolates of the EU1 lineage of Phytophthora ramorum, a fungus-like pathogen that causes extensive damage and mortality to a wide range of trees and other plants. Genomics Data 12, 17-21 (2017).

47. Martin, F. N., Bensasson, D., Tyler, B. M. \& Boore, J. L. Mitochondrial genome sequences and comparative genomics of Phytophthora ramorum and P. sojae. Current Genetics 51, 285-296 (2007).

48. Zheng, X. et al. A high-performance computing toolset for relatedness and principal component analysis of SNP data. Bioinformatics 28, 3326-3328 (2012).

49. Huson, D. H. \& Bryant, D. Application of phylogenetic networks in evolutionary studies. Molecular biology and evolution 23, 254-267 (2006).

50. Pembleton, L. W., Cogan, N. O. \& Forster, J. W. StAMPP: An R package for calculation of genetic differentiation and structure of mixed-ploidy level populations. Molecular ecology resources 13, 946952 (2013).

51. Blischak, P. D., Chifman, J., Wolfe, A. D. \& Kubatko, L. S. HyDe: a Python package for genome-scale hybridization detection. Systematic Biology 67, 821-829 (2018).

52. Patterson, M. et al. WhatsHap: weighted haplotype assembly for future-generation sequencing reads. Journal of Computational Biology 22, 498-509 (2015).

53. Stamatakis, A. RAxML version 8: a tool for phylogenetic analysis and post-analysis of large phylogenies. Bioinformatics 30, 1312-1313 (2014).

54. Cingolani, P. et al. A program for annotating and predicting the effects of single nucleotide polymorphisms, SnpEff: SNPs in the genome of Drosophila melanogaster strain w1118; iso-2; iso-3. Fly 6, 80-92 (2012).

\section{Figures}




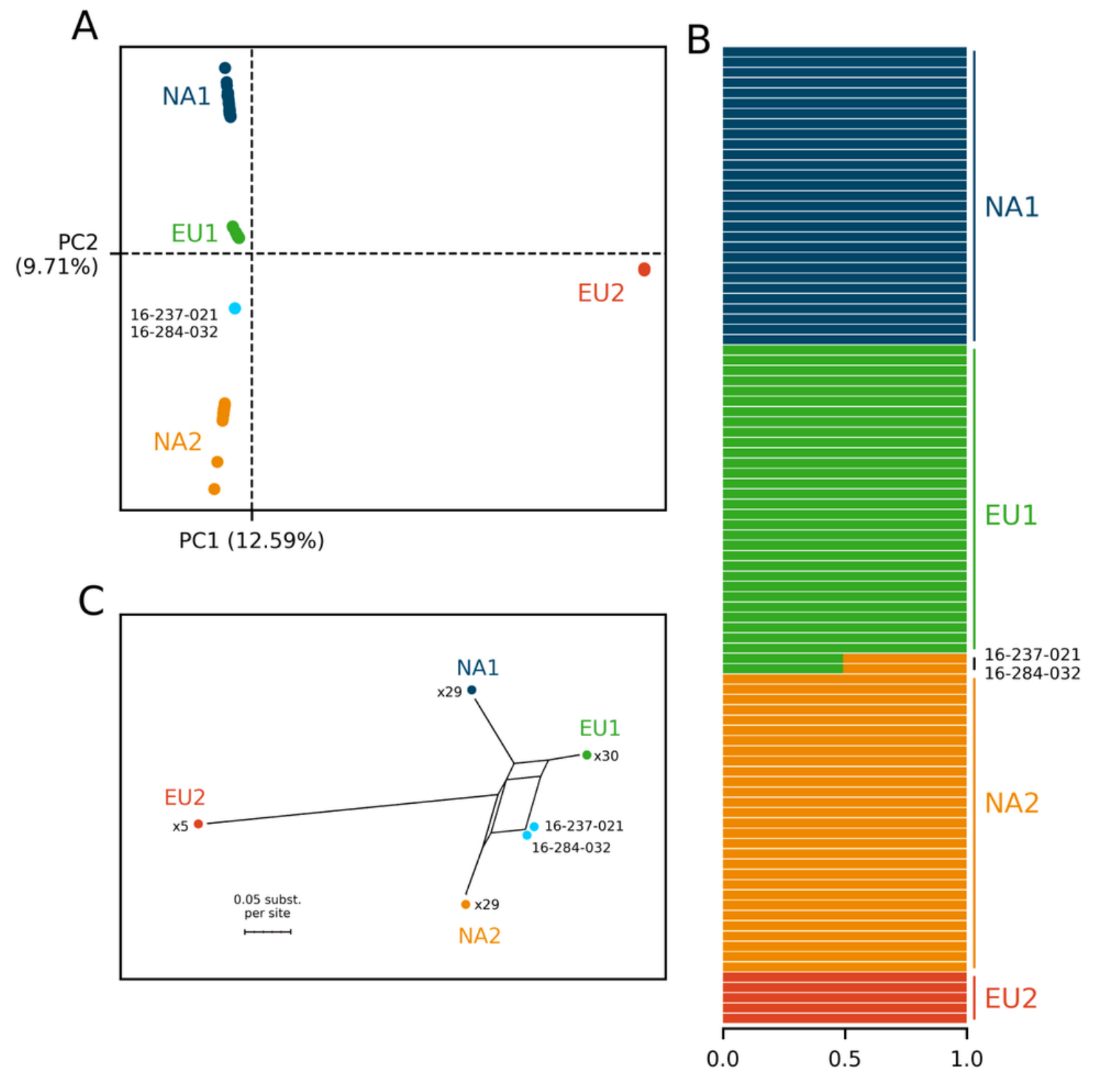

\section{Figure 1}

Hybridization between divergent clonal lineages EU1 and NA2 of Phytophthora ramorum. We analyzed 95 whole genomes of P. ramorum and extracted 450,656 single nucleotide polymorphisms (SNP) to characterize the populations; A) Population structure analysis of $P$. ramorum using a principal component analysis; each dot represents the genome of a P. ramorum isolate; B) Ancestry estimation using Admixture analysis of $\mathrm{P}$. ramorum lineages and putative hybrids at $\mathrm{K}=4$; each bar represents the genome of a P. ramorum isolate; $\mathrm{C}$ ) Neighbor-net phylogenetic network reconstructed from a matrix of pairwise Nei's genetic distances between isolates of P. ramorum. Samples 16-237-021 and 16-284-032 are the two putative hybrids. 


\section{A - Conserved hypothetical protein}

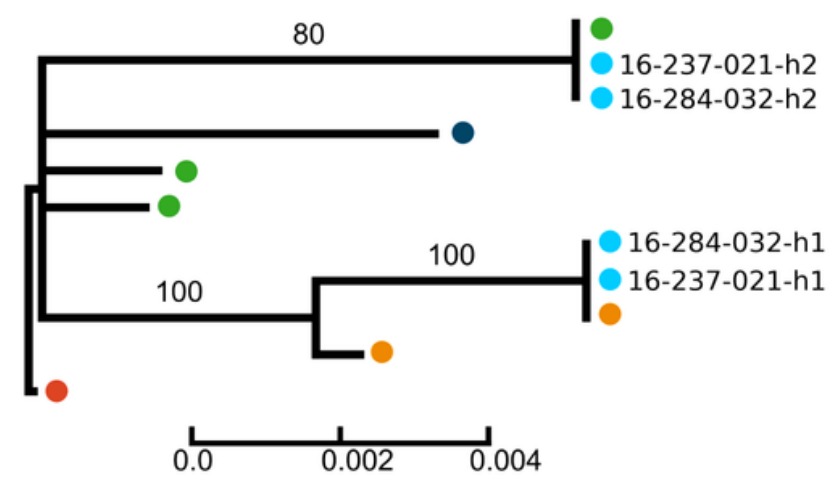

C - Cell 12A endoglucanase

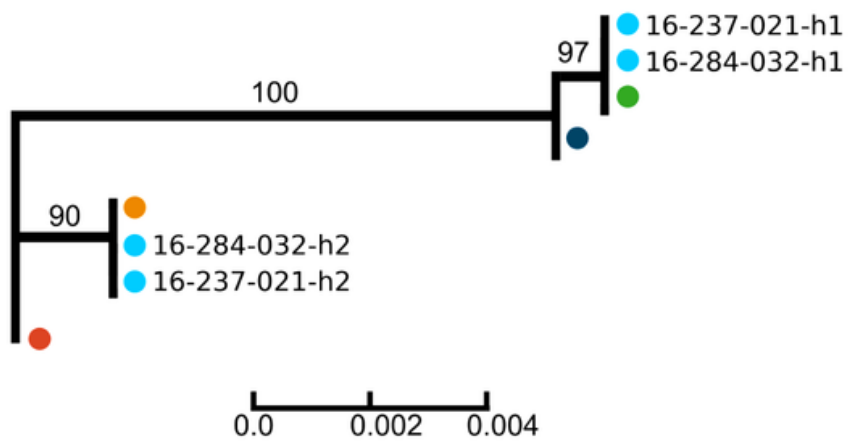

- EU1

- NA2
- NA1
B - Peroxisomal membrane protein

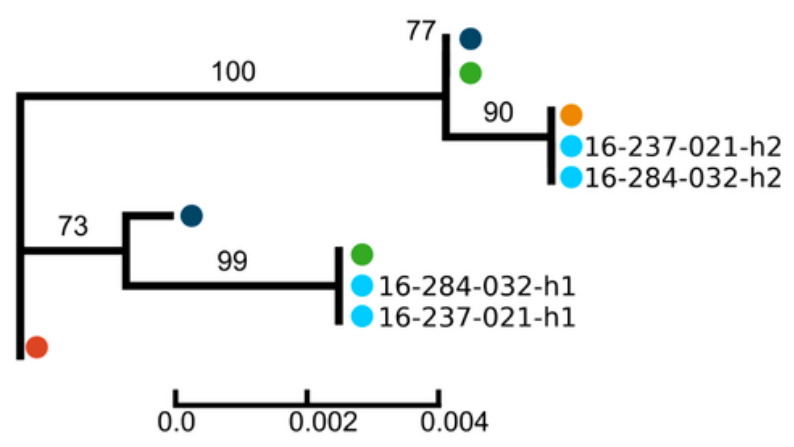

D - Mitochondrial DNA

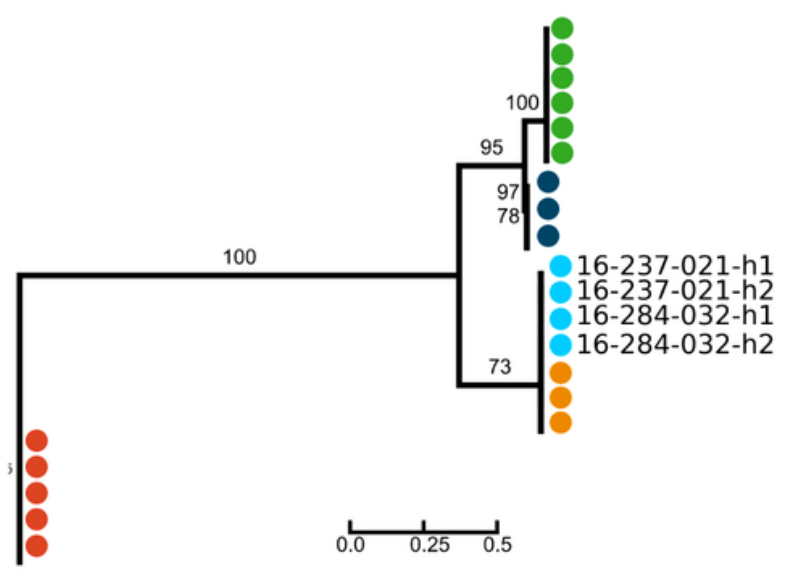

EU2
Hybrids

\section{Figure 2}

Haplotype phylogeny shows independent assortment of alleles at nuclear loci and uniparental inheritance of mitochondrial haplotype in the Phytophthora ramorum hybrid. Neighbor-joining tree of haplotypes of P. ramorum indicates meiotic recombination with nuclear phased haplotypes clustering with haplotypes of one of the two parents and mitochondrial haplotypes clustering with lineage NA2. Phased haplotypes were obtained by using short genome regions with physical phasing when two or more variants co-occur 
on the same sequencing read. A to C, nuclear genes; D, mitochondrial genome comprising 103 SNPs. For the two hybrid samples (16-237-021 and 16-284-032), the two haplotypes are indicated with h1 and h2.

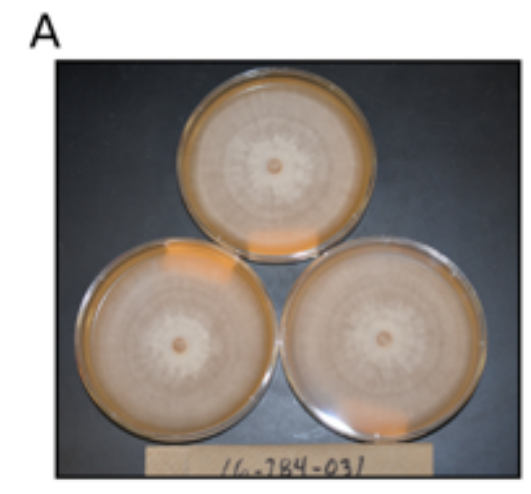

B

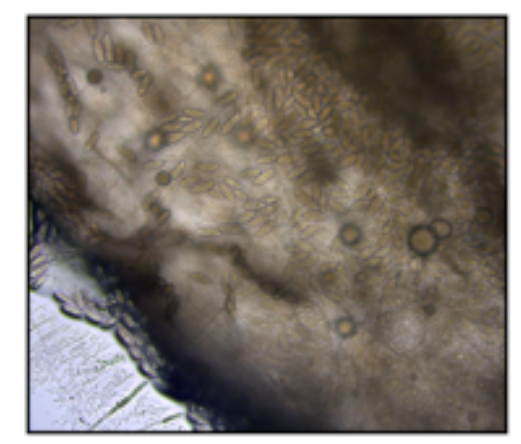

C

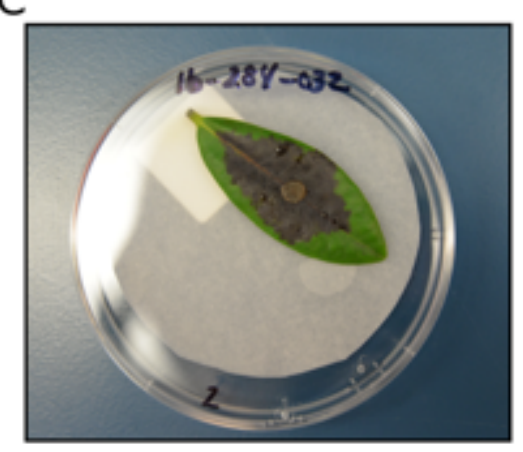

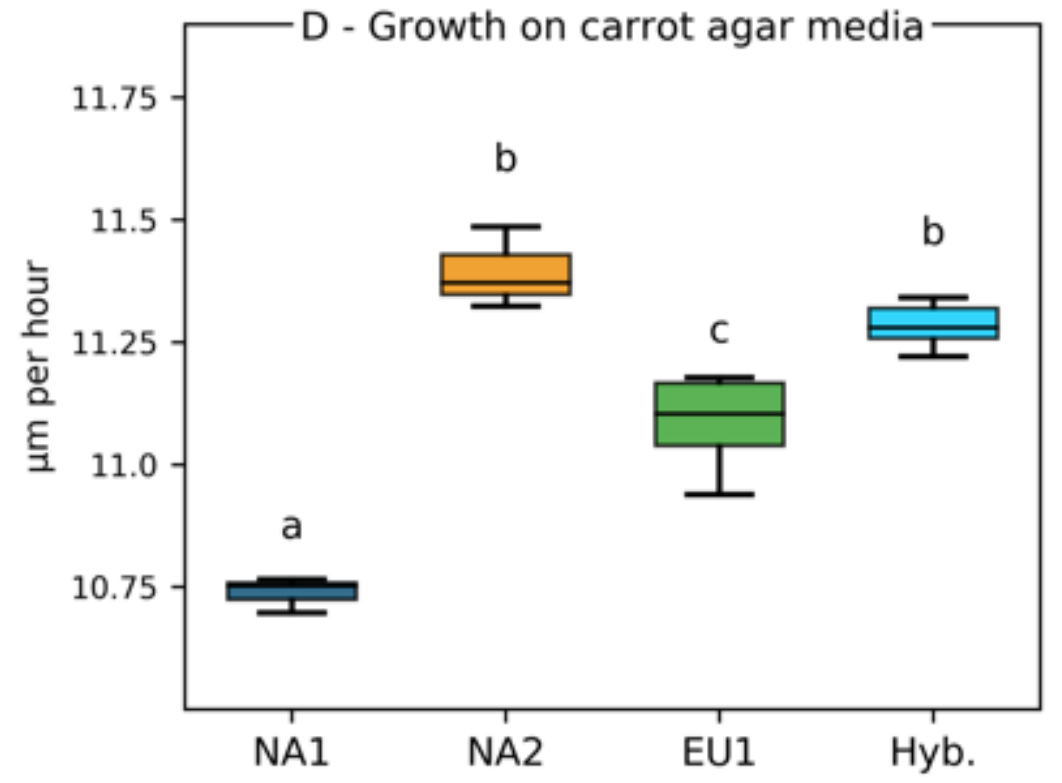

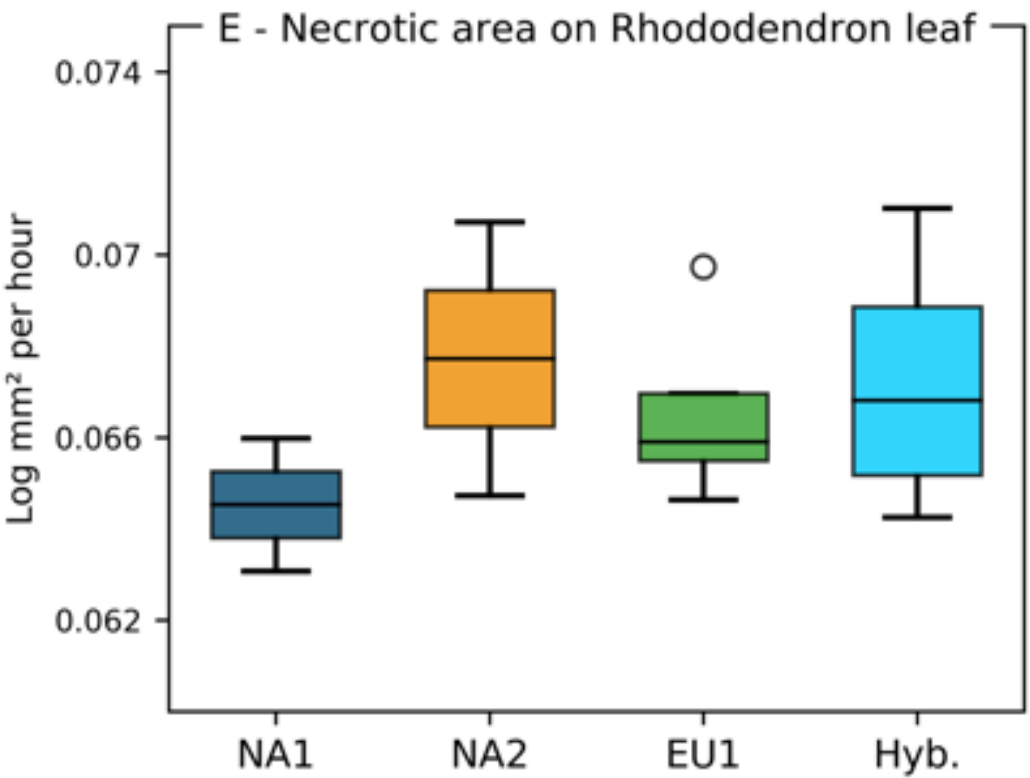

\section{Figure 3}

Phytophthora ramorum hybrid can sporulate, infect a host and has intermediate phenotypes. A) Morphology of P. ramorum hybrid isolate 16-284-032 growing on carrot agar, showing fluffy growth where sporangia are produced; $B$ ) Sporangia produced by the P. ramorum hybrid in culture; C) Rhododendron leaves infected by hybrid P. ramorum isolate 16-284-032; D) Growth of P. ramorum lineages and hybrids on carrot agar medium. Samples with different letters are statistically different (t-test at $p=0.05$ ); E) Growth of P. ramorum lineages and hybrids measured as necrotic area ( $\pi \times$ lesion length $\mathrm{x}$ width) over time on rhododendron leaves; there was no significant difference in lesion growth among the lineages and hybrids. 


\section{Supplementary Files}

This is a list of supplementary files associated with this preprint. Click to download.

- SupplementaryInformation07062021.docx 